MATEC Web of Conferences 28, 02006 (2015)

DOI: $10.1051 /$ matecconf $/ 20152802006$

(C) Owned by the authors, published by EDP Sciences, 2015

\title{
Scanning System Non-destructive Inspection of Weld Connections
}

\author{
Truts Alexander ${ }^{a}$, Titov Victor, Loshitskiy Petr \\ RSSC RTC, St. Petersburg, Russia
}

\begin{abstract}
In this paper a system for branch pipes welding connections is described. One of the important problems for lots of production facilities is defect detection in pipelines. Often the defect detection is both dangerous and a matter of high liability. In such case robotic system are preferable to substitute humans as they grant higher precision, unbiased analyzing, good repeatability, etc. The system presented here satisfies all the requirements while being equipped with modern sensors for non-destructive defectoscopy and simple yet effective and unified design.
\end{abstract}

\section{Introduction}

Evolution of modern technologies, complex algorithms and precise mechanics provide developers with great opportunities for automation of inspection operations. The necessity for such automation is dictated by requirements for accuracy and repeatability as well as for safety issues. Of particular interest are methods of nondestructive testing (NDT) which must also fit the above requirements. In NDT a lot of methods can be utilized: acoustic emission, electromagnetic testing, laser testing methods, leak testing, magnetic flux leakage, liquid penetrant testing, magnetic particle testing, neutron radiographic testing, radiographic testing, thermal/infrared testing, ultrasonic testing, vibration analysis and visual testing [1].

A number of systems created for tube or pipe inspection most of which utilize one or several of the aforementioned principles. They can be classified by different criterions: types of the sensors, principles of sensors positioning with respect to the tube (pipe) and the purposes of inspection (detection, detection and measurement, etc.).

The concern of this paper is mainly of principles of sensors positioning and the constructive solutions implementing them. The first two classes of the inspection devices are mobile $[5,8]$ and stationary $[6,7]$. The mobile in turn can be sub classified on the inner- and outer-surface scanning systems (the former moves on the inside surface of the tubes, the later - on the outside).

A new variation of mobile outer-surface robotic system for non-destructive defectoscopy is discussed in this paper.

\section{System Description and Development Issues}

The system in case is designed for inspection of branch pipe welding connections. The pipes material is primarily metal (stainless steel, for this particular project exploitation environment) but the scanning system itself can be used on a wide variety of materials. The only limitation is the ultrasonic sensors (used in the system) capability to operate on a given material (generally for concrete and metal the sensors shows sufficient accuracy [4]).

For the system in this paper two types of welding connections are in the scope of attention: one which connects two branch pipes along their axes and the other providing connection of two branch pipes at 90 degree angle.

The system functioning is as follows. First, a track is mounted on the branch pipe at specified distance from the inspection area. The design of the track provides required stiffness between the branch pipe and the mounted track and diminishes backlashes. To ensure the required reliable mounting the track is equipped with the mechanism that ensures some tolerance to the branch pipe geometry uncertainties. This is achieved with a uniform distribution of forces exposed by the track on the branch pipe external surface. The total time required for the track mounting is no more than 2 minutes. After that a scanning module is installed on the track. The rollers of the scanning module moves in the guiding groove of the track in cyclic motion along welding connection that gives the system good precision characteristics. The scanning module carries three ultrasonic sensors and a video camera. For the ultrasonic sensors to have good contact with the branch pipe surface a hydraulic line is embedded into the scanning module construction to supply couplant in the sensors contact area. The maximum time required for the scanning module installation is less than 2 minutes. The control module of the system includes a laptop and a remote control. The software of the laptop consists of two parts: the ultrasonic

\footnotetext{
${ }^{\text {a }}$ Corresponding author: a.truts@rtc.ru
} 
analysis, the visualization and motion control modules. The remote control can used to adjust the position of the scanning module on the track if necessary.

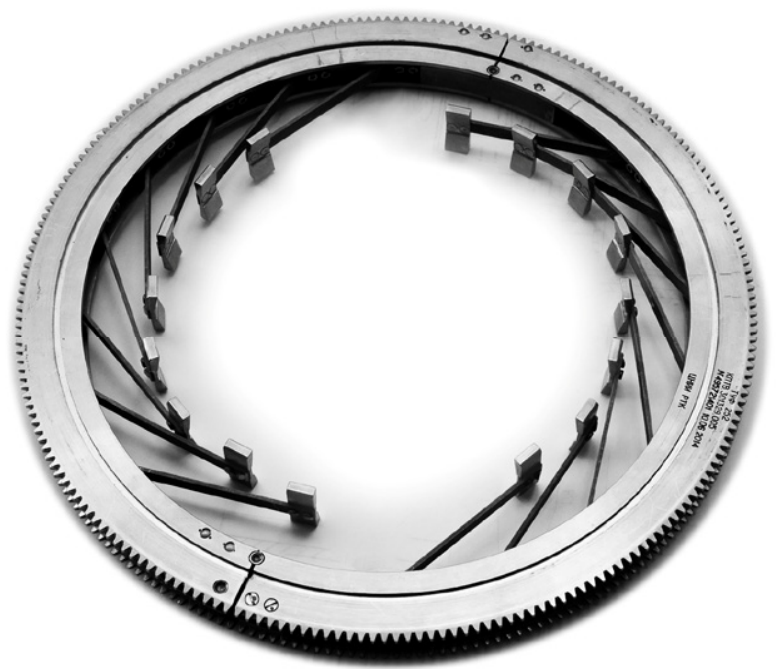

Figure 1. Tracking mechanism

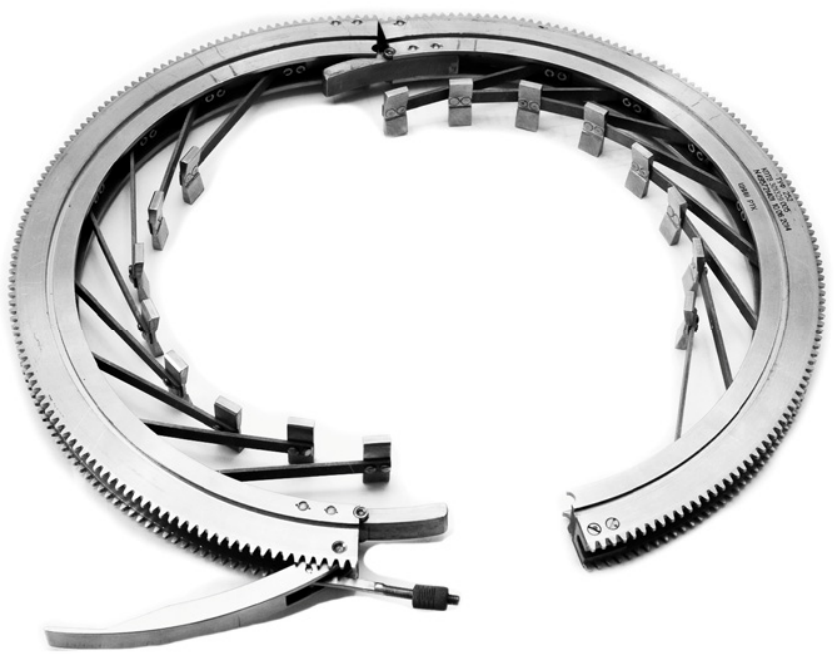

Figure 2 . Tracking mechanism (opened)

The track is shown in the Fig. 1. The track has toothing on its external radius that being put in contact with the gear ring of the scanning module grants the precise positioning and motion of the later The application of gearing is justified by its reliability, durability and no slippage in connection. The mechanism of the tack consists of two part joined with a special hinge that allow the track after unlocking to be opened at an angle slightly over $90^{\circ}$ (Fig. 2) Using a special hook two parts of the gear ring can be constricted and locked recovering the circle geometry of the track. Reliable mounting of the tack on the branch pipe is provided by special springs with supports (part of the track mechanism), which create necessary force for holding of the whole system on the branch pipe and compensate for the uncertainties of the branch pipe surface and diameter. In this case, tracking mechanism can be mounting on the branch pipe in a short time and locked on place near the welding connection. The springs are arranged in a ring array, are flexible and change the mounting force exerted by track on the branch pipe accounting for features of its surface structure. A series of the tracks of several diameters with unified designed is developed that allows the same scanning module to be used for all the branch pipe diameters. Despite the split construction of gear ring, the system expresses a high precision due to the using of centering rings, special catchers and applying the required force on the two-parts joint tracking mechanism.

The scanning module is shown in the Fig. 3 and Fig. 4. It includes electromechanical assembly which consists of a DC motor, planetary gear, encoder, and absolute encoder. On the output shaft of the electromechanical assembly a gear ring is fastened which roll on the track toothing. Special rollers fixate the scanning module on the track in all directions. The scanning module configuration can be adjusted by changing position of guiding arms that makes it possible to install it on the tracks of different diameters. With changing of the guiding arms position the positions of the ultrasonic sensors is also adjusted to be located on the branch pipe external surface. In addition to this the ultrasonic sensors are mounted on special guides that adjust the position of the sensor in radial and axial directions. In order to ensure stable contact during the motion the springs are installed on the guides that press down the sensor to the branch pipe surface.

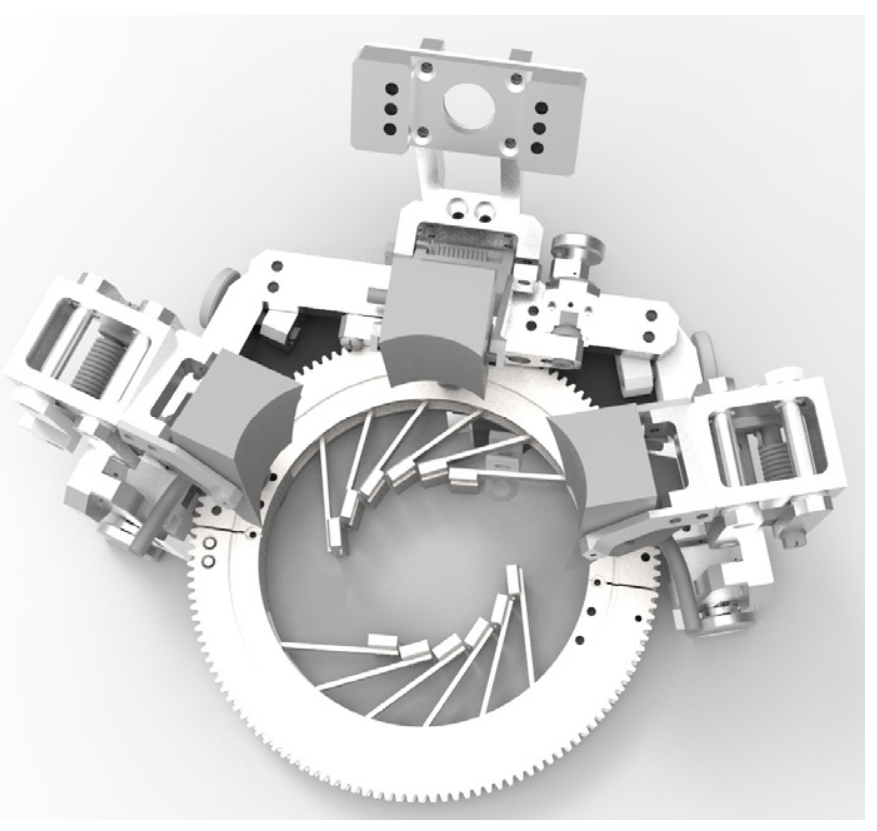

Figure 3. Scanning module (3D model)

Application of adaptive mechanics in the scanning module mechanism significantly reduces the price of the whole system as it requires only one scanning module to be produced and used for different inspection objects. That is yet another step to the system unification.

Acoustic contact and conductivity for this type of ultrasonic sensors are highly dependent on the surface quality and existence of gaps between the sliding surface of the sensor and the surface to be inspected. To minimize the effect of these the couplant is being 
supplied through hydraulic distributor during the scanning. The couplant supply rate is controlled to maximize the positive effect.

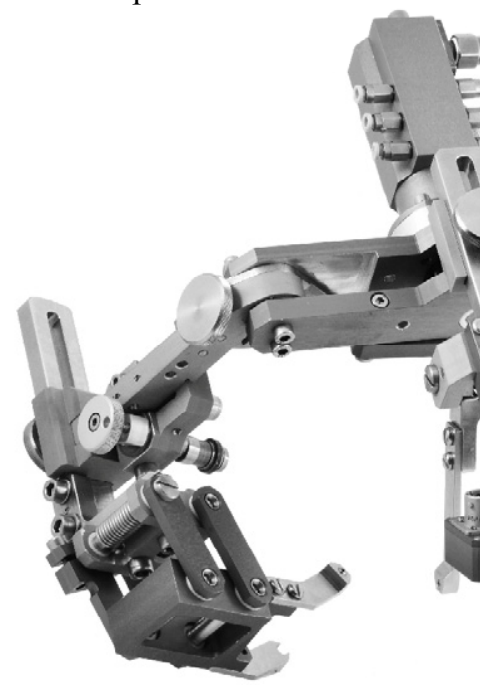

Figure 4. Scanning module

One of the cutting edge technologies in ultrasonic defectoscopy that leads to increase in both accuracy and amount of data obtained is ultrasonic phased arrays (UPA). UPA is a set of piezoelectric elements located on one plate. This technology is one of the most advanced and provides high quality of data ans low scanning time. The data obtained is multidimensional time series. The analysis of the data provides defectoscopist not with just capability of defect detection but also such defect parameters like its size and location in the material of the inspection object. can be estimated. Extra increase in the likelihood of defect detection is also achieved by spreading ultrasonic waves in material in different halfplanes. In the system such special separation is implemented by placing three sensors at different angles between them. Additionally the rear sensors are inclined toward each other in the plane perpendicular to the branch pipe axis.

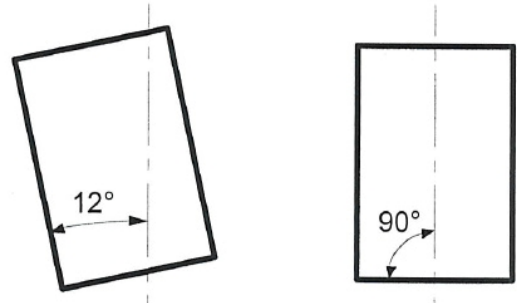

Figure 5. Placement of phased array sensors.

The workflow in the sense of a user involvement is presented in the system as two main modes of operation:

- manual;

- automotive;

In the automotive mode the inspection is conducted and supervised with the laptop software by the user via graphical user interface. In this mode after position calibration the scanning module takes full rotation about the branch pipe conducting scanning procedure and the defectoscopy data acquisition. The data is stored on the laptop hard drive for further processing and analysis. The data from ultrasonic sensors is coupled with the scanning module position on the track that makes it possible to restore the exact position of a defect on the branch pipe.

In the manual mode the position and velocity of the scanning module movements are controlled by the user in order to obtain more detailed information about specific area on the branch pipe surface or to verify the presence of the detected defect and/or its parameters. The movements of the scanning module can be controlled from both the laptop and the remote control.

\section{Conclusion}

In this paper a description of the system for inspection of branch pipes surface and welding connections was presented. The developed system has successfully passed all the tests at an experimental testbed and proved all declared characteristics. The system presented has a number of design features that give it advantage over the analogous systems:

- three ultrasonic phased array sensors providing good accuracy;

- universality of application in the domain: the system allow to inspect both butt and fillet welds of the branch pipes;

- adaptive mechanics of the scanning module makes the system applicable for a series of branch pipe diameters;

- $\quad$ fast mounting and installation procedures;

- optimal time of scanning process;

- $\quad$ high repeatability.

The factual values of some of the parameters are presented in Tab.1.

Table 1.

\begin{tabular}{|c|c|c|c|}
\hline & \multicolumn{2}{|c|}{ Parameter } & \multicolumn{2}{|c|}{ Value } \\
\cline { 2 - 4 } & $\begin{array}{c}\text { Maximal speed of the scanning } \\
\text { module in scanning process }\end{array}$ & {$[\mathrm{m} / \mathrm{min}]$} & $0-2.2$ \\
\hline & Track mounting time & {$[\mathrm{sec}]$} & 30 \\
\hline Range of branch pipe diameters & {$[\mathrm{m}]$} & $\geq 0,07$ \\
\hline & Track mass & {$[\mathrm{kg}]$} & $\geq 3$ \\
\hline & Scanning module mass & {$[\mathrm{kg}]$} & 4,5 \\
\cline { 2 - 4 } & Battery operating time & {$[\mathrm{hours}]$} & 4 \\
\hline
\end{tabular}

The experience shows that the developed system provides great opportunities for the branch pipe welding connections inspection process automatization, reduction of human factor influence on the process of measurement and the data acquired. The design and characteristics establish its multipurpose application and in-domain universality that helps to fulfill the main goal of such 
systems creation - to substitute humans in unfriendly and dangerous environment.

The Applied scientific investigations are carried out with the financial support of the Russian Federation represented by the Russian Ministry of Education and Science. The unique identifier of the Applied scientific investigations is RFMEFI57814X0046.

\section{References}

1. Information on https://www.asnt.org/MinorSiteSections/AboutASN T/Intro-to-NDT

2. Annila L. Non-destructive testing of pipelines. Information on http://st-div.web.cern.ch/stdiv/workshop/ST2001WS/Proceedings/Poster_3101/ Annila.pdf
3. Information on http://www.puretechltd.com/services/robotics/

4. GUIDEBOOK ON NON-DESTRUCTIVE TESTING OF CONCRETE STRUCTURES IAEA, VIENNA, 2002 IAEA-TCS-17, ISSN 1018-5518

5. Information on http://www.puretechltd.com/services/robotics/

6. Information on http://www.newtechsystems.com/TubePRO_554PB. $\underline{\mathrm{htm}}$

7. Information on http://ndtsupply.com/pitco-ndt-pipeinspection-equipment.html

8. Kim S., Chang Hoi Kim, Bae Y, Na H., Jung S. NDT inspection mobile robot with spiral driven mechanism in pipes.// Robotics (ISR), 2013 44th International Symposium on Robotics (ISR), (2013) - PP. 1 